\title{
Gastric Outlet Obstruction in a Cystic Fibrosis Patient
}

\author{
Zubair Khan $\mathbf{M}^{*}$, Ahamd $\mathbf{W}^{2}$, Patel $\mathrm{K}^{1}$ and \\ Chaudary $\mathbf{N}^{1}$ \\ ${ }^{1}$ Department of Internal Medicine, Virginia \\ Commonwealth University Hospital, USA \\ ${ }^{2}$ Khyber Teaching Hospital, Pakistan \\ *Corresponding author: Muhamamd Zubair \\ Khan, Department of Internal Medicine, Virginia \\ Commonwealth University Hospital, USA
}

Received: December 26, 2020; Accepted: January 07, 2021; Published: J anuary 14, 2021

\begin{abstract}
Eosinophilic gastritis is an uncommon disease characterized by focal or diffuse eosinophilic infiltration of the gastric wall and is usually associated with dyspepsia and peripheral eosinophilia. The stomach and small bowel are usually involved in Eosinophilic Gastrointestinal Disorder (EGID) which is called Eosinophilic Gastroenteritis (EG), but the esophagus and colon are rarely involved. Gastrointestinal obstruction rarely occurs with eosinophilic gastroenteritis and usually happens when the eosinophils predominantly involve the muscular layer of the gastrointestinal tract. Here we present a case of a Cystic Fibrosis (CF) patient in which Gastric Outlet Obstruction (GOO) is caused by eosinophilic gastritis and treated successfully with steroids.
\end{abstract}

Keywords: Cystic fibrosis, Eosinophilic gastroenteritis

\section{Introduction}

Primary eosinophilic gastrointestinal disorders (e.g. Eosinophilic Esophagitis (EoE), eosinophilic gastritis, Eosinophilic Gastroenteritis (EG), eosinophilic enteritis, and eosinophilic colitis) are defined as disorders in which the wall of the gastrointestinal tract becomes filled with large numbers of eosinophils in the absence of other potential causes of eosinophilia. Eosinophilic gastroenteritis is a rare cause of abdominal pain of unknown etiology [1]. Infiltration of the muscular layer of the gastrointestinal tract in eosinophilic gastroenteritis can cause small bowel obstruction and Gastric Outlet Obstruction (GOO) but GOO is extremely rare [2]. Here we present a case of GOO in a cystic fibrosis patient which is due to eosinophilic gastroenteritis.

\section{Case Report}

A fifty-four-year-old female with past medical history of cystic fibrosis, Gastroesophageal Reflux Disease (GERD), hysterectomy, iron deficiency and migraine presented to an outside hospital with a 24-hour history of severe epigastric pain and a 1-week history of nausea and vomiting, flatulence, and indigestion. She was found to have acute pancreatitis and was admitted to the hospital. Vital signs were normal. Physical exam was unremarkable. Her labs were unremarkable for hepatitis, cholecystitis, and urinary infection. Chest $\mathrm{x}$-ray ruled out pneumonia, abdominal plain film ruled out ileus, and a right upper quadrant ultrasound did not show gallstones. The patient continued to complain of abdominal pain despite improvements in her serum amylase and lipase. A Computed Tomography (CT) scan of the abdomen and pelvis revealed distal esophageal thickening and a dilated stomach. After four days of hospitalization she was discharged home tolerating a regular diet with improvement in her nausea and vomiting.

At 1-week outpatient follow-up, she continued to have nausea and vomiting with intermittent abdominal pain. Evaluation included fluoroscopy esophagram which revealed normal esophagus, gastroparesis and gastric outlet obstruction. MRI abdomen showed liver cysts. The nuclear gastric emptying study confirmed gastroparesis with 4-hour gastric retention of $37 \%$. She underwent Upper Gastrointestinal Endoscopy (UGI) and colonoscopy (LGI).
LGI showed melanosis cold diffusely. UGI showed multiple furrows in the distal $10 \mathrm{~cm}$ of the esophagus, diffuse food, liquid in the stomach, and severe pre-pyloric stenosis causing obstruction without mass lesion or ulceration. Biopsies of the mid-esophagus, distal esophagus, gastric and pyloric stenosis revealed $>40-60$ (Eos/HPF) confirming EG. She underwent serial dilation of pyloric stenosis and failed to show improvement. Repeat fluoroscopic esophagram with small bowel follow through and a nuclear gastric emptying study showed persistent GOO and gastroparesis. The combined results were due to eosinophil infiltration of the pre-pyloric muscular layer causing GOO. The patient was started on metoclopramide $10 \mathrm{mg}$ po q.i.d, omeprazole $40 \mathrm{mg}$ po b.i.d and oral viscous budesonide slurry $(1 \mathrm{mg} / 2 \mathrm{ml})$ b.i.d. At follow up 3 months later she had complete resolution of nausea, abdominal pain, and weight loss. The patient tolerated the medications well. She is consuming a regular diet and was successfully managed without surgical correction for GOO.

\section{Discussion}

\section{The switch between EGID, EoE, and EG is confusing}

In this case, we present a patient with known CF and concomitant non-specific GI symptoms. Even after her serum amylase, lipase improved, the patient continued to have abdominal pain, which was highly suspicious for malignancy and EGID. The diagnosis of EGID and malignancy was not clinically suspected prior to obtaining the endoscopy and biopsy results, which underscores the importance of this additional work-up. To our knowledge few cases have been presented in the literature about the co-existence of EoE and CF [3] but not EoE with gastric outlet obstruction. We presented this case report about EG with gastric outlet obstruction in CF patients and educated the CF specialist how to treat such kind of patients [3].

The pathogenic mechanism of EG is poorly understood but it is thought that a small amount of eosinophils in the GI tract mucosa play a key role. The studies suggest that allergens may cross the intestinal mucosa and prompt the $\mathrm{TH} 2$ response where IL-13 and IL-5 stimulate the eosinophils to produce eotaxin-3, which recruit additional eosinophils into the GI tract. And may lead to decrease the barrier [3]. Clinically, atopic diseases such as allergic rhinitis, food allergies, atopic dermatitis, and asthma are commonly associated with 
EG. The pathogenesis of CF does not overlap with that of EG and food allergies have rarely been reported in CF patients. The association of EGID with $\mathrm{CF}$ is unlikely because $\mathrm{CF}$ is purely a genetic disease while EGID is allergen-mediated disease and genetics are less likely to play a role in this disease. The most common gene involved in the EGID (EoE) is the CCL-26 and TSLP [4] but in CF, the 508 gene is usually involved. Although these facts show that an association is unlikely but the the EGID with CF has been mentioned in other studies which seems that the pathogenesis of EGID with CF is not fully understood and requires additional study. The EGID (EG) association has been reported in PPI-responsive esophageal eosinophilia, Celiac disease, Crohn's disease, infection, Hyper eosinophilic syndrome, achalasia, drug hypersensitivity, vasculitis, Pemphigus, connective tissue diseases, and graft vs. host disease.

The most common cause of GOO is not? due to peptic ulcer disease but due to PPI and other effective therapy. Malignancy and EG have more recently emerged as important causes of GOO. EG and malignancy should be ruled out in all GOO patients. The other common causes of GOO that should be considered in the differential diagnosis include post-surgical strictures, pancreatic pseudocyst, chronic pancreatitis, gallstones, and Crohn's disease [5]. Sometimes in children, EG has similar symptoms and morphology to congenital pyloric stenosis. Malignancy is highly suspected among patients who have weight loss and who are greater than 50 years old. A thorough history, physical examination, radiological films, and tumor markers are all greatly helpful to rule out a malignancy.

Eosinophilic gastroenteritis patients may have acute abdomen due to intestinal obstruction, acute pancreatitis, perforation, and intussusception. Only a few cases of EG present with acute pancreatitis of unknown etiology, diagnosed with biopsy, and are subsequently treated with steroids without recurrence. In our case, the patient's pancreatitis may be due to CF or eosinophilic infiltration. The patient's symptoms resolved with conservative treatment and had no recurrence which showed that pancreatitis was probably due to eosinophilic infiltration [6].
There are 3 general categories of treatment for EGID, often referred to as "the three D's": drugs, diet, and dilation. Corticosteroids used with dietary intervention are a mainstay treatment and the firstline agents in many cases. In our case, the patient was given an oral steroid and for GOO the pylorus was dilated several times to relieve symptoms. The patient's symptoms were fully resolved.

\section{Conclusion}

The association of CF and EGID (eosinophilic gastritis with esophagitis) is highly unlikely because CF is a purely genetic disease, whereas EGID is allergen-mediated disease and genetics are less likely to play a role in this disease. In previous case reports, patients with eosinophilic gastritis with GOO were treated surgically or medically, depending on the condition. In our case, the patient was treated with steroids and completely recovered from GOO without surgery. Thus, it is plausible that both CF and non-CF patients who have eosinophilic gastritis with GOO can benefit from similar treatment. Moreover, adult patients with symptoms of GOO require endoscopic, histological, and radiological investigations to make the correct diagnosis.

\section{References}

1. Khan S. Eosinophilic gastroenteritis. Best Pract Res Clin Gastroenterol. 2005; 19: 177-198

2. Chen MJ, Chu CH, Lin SC, Shih SC, Wang TE. Eosinophilic gastroenteritis: clinical experience with 15 patients. World J Gastroenterol. 2003; 9: 2813 2816.

3. Goralski JL, Lercher DM, Davis SD, Dellon ES. Eosinophilic esophagitis in cystic fibrosis: a case series and review of the literature. J Cyst Fibros. 2013; 12: $9-14$

4. Sherrill JD, Rothenberg ME. Genetic and epigenetic underpinnings of eosinophilic esophagitis. Gastroenterol Clin North Am. 2014; 43: 269-280.

5. Mohammed AA, Benmousa A, Almeghaiseeb I, Alkarawi M. Gastric outlet obstruction. Hepatogastroenterology. 2007; 54: 2415-2420.

6. Sheikh RA, Prindiville TP, Pecha RE, Ruebner BH. Unusual presentations of eosinophilic gastroenteritis: case series and review of literature. World $J$ Gastroenterol. 2009; 15: 2156-2161. 